\title{
Esophageal Lichen Planus Stricture
}

\author{
Farhad Zamani ${ }^{1,}$, , Mehran Haghighi ${ }^{1}$, Mohammad Roshani ${ }^{1}$, Masoudreza Sohrabi ${ }^{1}$
}

1. Gastroenterology and Liver Disease Research Center, Iran University of Medical Sciences, Firoozgar Hospital, Tehran, Iran

\section{* Corresponding Author:}

Farhad Zamani, MD

GastroIntestinal and Liver Diseases

Research Center ,Firoozgar Hospital,

Behafarin Ave. Valiasr Sq. Tehran,

159374, Iran

Telefax: + 982188941831

Email: zamani.farhad@gmail.com

Received: 11 Jul. 2018

Accepted: 11 Nov. 2018

\section{ABSTRACT}

Lichen planus is a dermatologic chronic inflammatory disease that usually involved the skin and mucosa. Esophageal lichen planus with stricture is rare presentation of this disease. It is usually associated with oral mucosal involvement and commonly presenting in middle-age female. In present case, we describe a 48 -year-old woman with history of oral lichen planus who referred to us for progressive dysphagia for solid food. She had not history of other causes of dysphagia. Upper endoscopy revealed a benign stricture located in proximal of esophagus that comparable with lichen planus features. The patient underwent three sessions of wireguided bougie dilation that improved her symptoms totally. Lichen planus has to be considered in differential diagnosis in patients with dysphagia.

\section{KEYWORDS}

Dysphagia, Esophageal stricture, Lichen planus

Please cite this paper as:

Zamani F, Haghighi M, Roshani M, Sohrabi MR. Esophageal Lichen Planus Stricture Middle East J Dig Dis 2019;11:52-54. doi: 10.15171/mejdd.2018.128.

\section{INTRODUCTION}

Apart from malignant involvement of esophagus, a number of benign conditions such as gastroesophageal reflux disease, ingestion of caustic agents, and radiotherapy can cause esophageal stricture. Some mucocutaneus diseases can also involve the esophagus and cause strictures, including epidermolysis bullosa, pemphigoid, pemphigus vulgaris, and lichen planus. ${ }^{1}$

Lichen planus (LP) is an inflammatory disorder with probable autoimmune pathology, which affects squamous mucosa as well as skin. The skin lesions commonly emerge as violaceous papules of the wrists, low back, and ankles. Mucosa of mouth and genitalia may get involved and possibly with no evidence of skin lesions. ${ }^{1}$ Esophageal LP (ELP) is a rare and under-recognized manifestation of lichen plaque. Due to the rarity and unfamiliarity of the disease, it is not uncommon to take few to several years to achieve the correct diagnosis and proper management. ${ }^{2}$ We present a case of a severe esophageal stricture due to mucosal LP.

\section{CASE REPORT}

A 48-year-old woman with a medical history of biopsy-proven oral LP diagnosed 5 years earlier was presented for an evaluation of pro- 


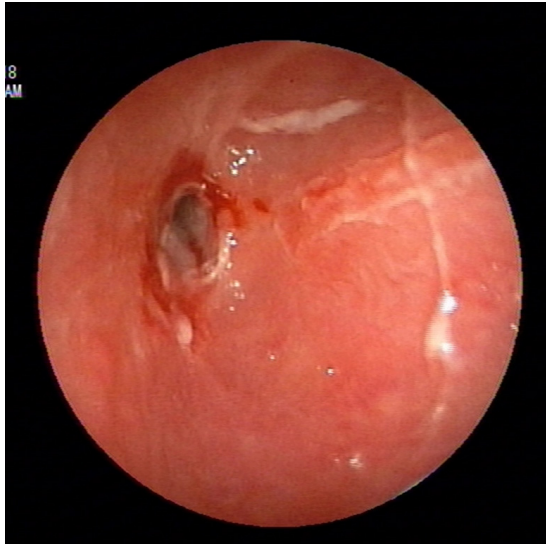

Fig.1: Stricture in the upper third of esophagus with inflamed mucosa, pseudomembranous formation, and ulceration

gressively increasing dysphagia for solid foods for the past 6 months. She did not have typical heartburn or regurgitation symptoms consistent with reflux disease. There was no history of corrosive injury or radiation to esophagus. She was on prednisone $5 \mathrm{mg}$ /day, omeprazole $20 \mathrm{mg} /$ day, and azathioprine $50 \mathrm{mg} /$ day and there was not any active lesion at buccal mucosa at the time of the last visit.

Esophagogastroduodenoscopy (EGD) was performed, which showed proximal esophageal benign appearing stricture $20 \mathrm{~cm}$ from the incisors (figure 1). There was peeling of the mucosa away from the esophagus leaving a friable, inflamed surface that bled easily on contact. Since the scope could not be negotiated distally, a pediatric scope was used. It was a short segment stricture and distal gastrointestinal (GI) tract was normal. The endoscopic and clinical findings were consistent with ELP, therefore esophageal biopsy sample was not taken. Due to the severity of the symptoms, the patient underwent three sessions of wire-guided bougie dilation up to $39 \mathrm{Fr}$. (figure 2) and her symptoms improved.

\section{DISCUSSION}

Esophageal stricture secondary to LP is a very rare condition. ${ }^{1}$ Although, asymptomatic esophageal involvement was reported in up to $25 \%$ of patients with $\mathrm{LP},{ }^{3}$ less than $1 \%$ of the patients with oral LP develop symptomatic esophageal strictures. ${ }^{4} \mathrm{LP}$ itself constitutes $0.1-1.2 \%$ of all skin disorders. ${ }^{5}$

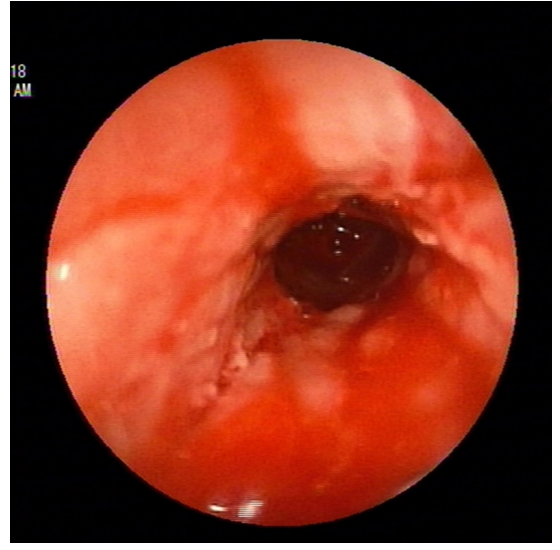

Fig.2: Proximal stricture treated with dilatation.

The disorder predominantly involves middle-aged to elderly women (approximately $87 \%$ of women with a median age of 61.9 years. ${ }^{6}$ Dysphagia is the most common symptom followed by odynophagia and food impaction. ${ }^{4,6}$ EGD plays a diagnostic and therapeutic role in these patients and should be performed in all patients with LP and GI symptoms. Macroscopic findings are non-specific but include ulcers, erosions, stricture formation, whitish papules, esophageal pseudomembranes as well as inflamed and friable mucosa. ${ }^{1,4,6}$ The findings may be mistaken for reflux esophagitis, eosinophilic esophagitis, or candida esophagitis. ${ }^{4}$ Most patients with LP were already on steroids and immunosuppressive medications, which make the diagnosis of candidiasis even more probable. In the case of uncertainty biopsy should be performed. Strictures in ELP occur more commonly in the proximal esophagus, ${ }^{4}$ sparing the gastroesophageal junction, unlike reflux esophagitis. ${ }^{1}$ Histopathology may be non-diagnostic in half of the cases with interpretations of esophagitis or chronic inflammation. ${ }^{6}$ Findings that help support a diagnosis of ELP include a dense band-like lymphocytic infiltrate involving the lamina propria, basal layer degeneration, and apoptotic basal keratinocytes (Civatte bodies). ${ }^{4,6}$ Even the submucosal lymphocytic infiltration though consistent with LP, is non-specific and may be seen in patients on some drugs like thiazides or anti-malarial agents, in infectious or pill-induced esophagitis, and occasionally in gastroesophageal reflux disease. ${ }^{1}$ Oral LP has a very low malignant potential of less than $1 \%{ }^{1}$ ELP may also 
be associated with esophageal squamous cell carcinoma (SCC). There are four reported cases of ELP progressing to esophageal SCC in the literature. ${ }^{4}$

Because of rarity, diagnosis of ELP is often challenging and sometimes multiple endoscopies are performed before reaching the diagnosis. ${ }^{6}$ The first step in the treatment of ELP is conservative management using topical or systemic medications. Therefore, administration of corticosteroids, retinoids, and griseofulvin have been reported. ${ }^{1}$

Reports have shown good responses with tacrolimus, cyclosporine, and intralesional triamcinolone acetate for improving the symptoms. ${ }^{7-9}$ In patients who remain symptomatic despite medical management, endoscopic dilation is a feasible option. In this setting Koebner phenomenon (the formation of LP lesions in uninvolved site after trauma) at the sites of trauma with dilatation have been reported in some papers. ${ }^{6,4}$ On the other hand dilatation is indispensable once stricture has developed in order to achieve immediate improvement in symptoms. ${ }^{6}$ Moreover, some patients may even need frequent dilatation procedures or ultimately definitive surgical management. ${ }^{6}$ Recently, esophageal metal stent has been applied successfully and considered as an option once standard medical therapy and endoscopic dilation fails. ${ }^{10}$

In conclusion, LP of esophagus should be considered in differential diagnosis for dysphagia, especially for middle- to elderly-aged women, with refractory proximal stricturing disease, and with concomitant mucocutaneus lesions.

\section{ETHICAL APPROVAL}

There is nothing to be declared.

\section{CONFLICT OF INTEREST}

The authors declare no conflict of interest related to this work.

\section{REFERENCES}

1. Madhusudhan KS, Sharma R. Esophageal Lichen Planus: A Case Report and Review of Literature. Indian J Dermatol 2008;53:26-7. doi:10.4103/00195154.39738 .

2. Le Cleach L, Chosidow O. Clinical practice. Lichen planus. N Engl JMed 2012;366:723-32. doi:10.1056/ NEJMcp1103641.
3. Dickens CM, Heseltine D, Walton S, Bennett JR. The esophagus in lichen planus: An endoscopic study. $B M J$ 1990;300:84. doi:10.1136/bmj.300.6717.84.

4. Franco DL, Islam SR, Lam-Himlin DM, Fleischer DE, Pasha SF. Presentation, Diagnosis, and Management of Esophageal Lichen Planus: A Series of Six Cases. Case Rep Gastroenterol 2015;9:253-60. doi:10.1159/000437292.

5. Al-Shihabi BM, Jackson JM. Dysphagia due to pharyngeal and oesophageal lichen planus. $J$ Laryngol Otol 1982;96:567-71. doi:10.1017/ S0022215100092835.

6. Rao B, Gulati A, Jobe B, Thakkar S. Esophageal Lichen Planus: Understanding a Potentially Severe Stricturing Disease. Case Rep Gastrointest Med 2017;2017:5480562. doi:10.1155/2017/5480562.

7. Donnellan F, Swan MP, May GR, Kandel G, Marcon NE, Kortan PP. Fluticasone propionate for treatment of esophageal lichen planus. A case series. Dis Esophagus 2011;24:211-4. doi:10.1111/j.14422050.2010.01120.x.

8. Keate RF, Williams JW, Connolly SM. Lichen planus esophagitis: report of three patients treated with oral tacrolimus or intraesophageal corticosteroid injections or both. Dis Esophagus 2003;16:47-53. doi:10.1046/j.1442-2050.2003.00289.x.

9. Chaklader M, Morris-Larkin C, Gulliver W, McGrath $\mathrm{J}$. Cyclosporine in the management of esophageal lichen planus. Can J Gastroenterol 2009;23:686-8. doi:10.1155/2009/802075.

10. Stein P, Brun A, Zaidi H, Sejpal DV, Trindade AJ. Successful Treatment of a Persistent Esophageal Lichen Planus Stricture With a Fully Covered Metal Stent. ACG Case Rep J 2016;3:98-100. doi:10.14309/ crj.2016.12. 